\title{
Current state of transcatheter tricuspid valve repair
}

\author{
Dhaval Kolte, Sammy Elmariah
}

Division of Cardiology, Massachusetts General Hospital and Harvard Medical School, Boston, MA, USA

Contributions: (I) Conception and design: All authors; (II) Administrative support: All authors; (III) Provision of study materials or patients: All authors; (IV) Collection and assembly of data: All authors; (V) Data analysis and interpretation: All authors; (VI) Manuscript writing: All authors; (VII) Final approval of manuscript: All authors.

Correspondence to: Sammy Elmariah, MD, MPH. Cardiology Division, Department of Medicine, Massachusetts General Hospital, Harvard Medical School, 55 Fruit Street, GRB 800, Boston, MA 02114, USA. Email: selmariah@mgh.harvard.edu.

\begin{abstract}
Tricuspid regurgitation (TR) is a common valvular heart disease affecting $>1.6$ million people in the United States (US) and >70 million people worldwide. The age- and sex-adjusted prevalence of more than or equal to moderate TR in the US is estimated to be $0.55 \%$. One-year mortality increases with increasing severity of TR. Yet, the majority of patients with severe TR are managed medically in the absence of another indication for cardiac surgery, and isolated tricuspid valve (TV) surgery remains infrequent. To address this unmet clinical need, various transcatheter TV therapies are now being developed as an alternative to surgery in extreme- and high-risk patients with severe functional TR. Transcatheter TV repair devices are aimed at improving leaflet coaptation either directly by bringing the leaflets together (leaflet/ coaptation devices) or indirectly by repairing the dilated annulus (annuloplasty devices). In this review, we describe the current state of transcatheter TV repair therapies and summarize the available data on the efficacy and safety of various devices. Procedural and clinical outcomes of transcatheter TV repair therapies are expected to improve in the coming years with technological advancement, newer device iterations, and increased experience in this field. Appropriate patient selection, optimal timing of intervention, and evaluation of long-term outcomes and device durability will be key in ongoing and future studies.
\end{abstract}

Keywords: Tricuspid regurgitation (TR); tricuspid insufficiency; transcatheter tricuspid valve repair (transcatheter TV repair); tricuspid valve (TV)

Submitted Jun 12, 2019. Accepted for publication Sep 09, 2019.

doi: $10.21037 / \mathrm{cdt} .2019 .09 .11$

View this article at: http://dx.doi.org/10.21037/cdt.2019.09.11

\section{Introduction}

Tricuspid regurgitation (TR) is a commonly encountered valvular heart disease affecting $>1.6$ million people in the United States (US) and $>70$ million people worldwide $(1,2)$. Approximately $90 \%$ of TR in adults is functional (secondary) and can be due to left-sided myocardial or valvular disease, pulmonary vascular disease, right ventricular myopathy, chronic right ventricular volume overload, or idiopathic. Recently, using data from Olmsted County, the ageand sex-adjusted prevalence of more than or equal to moderate TR in the US was estimated to be $0.55 \%$ (3). The prevalence of TR increases with age and is significantly higher in women $v s$. men. The majority of patients have trivial or mild TR, which may be non-pathological. However, 1-year mortality increases with increasing severity of TR, reaching $36.1 \%$ in patients with severe TR (4).

Despite the high prevalence of TR and its association with increased mortality, $<8,000$ tricuspid valve (TV) surgeries (repair or replacement) are performed annually, and the majority of patients are managed medically in the absence of another indication for cardiac surgery (5). The in-hospital mortality rate after isolated TV surgery is $2 \%$ to $10 \%$ and there are limited data demonstrating improved survival (6-8). Isolated TV surgery, therefore, is rarely performed ( $\sim 500$ per year) (9). Hence, there is an unmet clinical need for novel surgical or transcatheter therapies for TR. In this article, we provide an overview of the current 
state of transcatheter TV repair therapies.

\section{TV anatomy-considerations for transcatheter TV repair}

The TV consists of 3 leaflets (anterior, posterior, and septal) arising from the tricuspid annulus (TA) and attached via chordae tendineae to the papillary muscles of the right ventricle (RV) (10). Similar to the saddle-shaped mitral annulus, the TA is also a nonplanar structurethe posteroseptal portion is more ventricular and the anteroseptal portion is more atrial—with a more flattened oval shape. The TA is also a dynamic structure, the shape and size of which varies throughout the cardiac cycle and with loading conditions. Normal tricuspid annular circumference and area in healthy subjects is $12 \pm 1 \mathrm{~cm}$ and $11 \pm 2 \mathrm{~cm}^{2}$, respectively, as measured by $3 \mathrm{D}$ echocardiography.

Three important structures lie in close proximity to the TV (11). First, the noncoronary sinus of Valsalva is adjacent to the anteroseptal commissure. Thus, transcatheter TV repair devices that require anchoring in this region pose the risk of aortic perforation. Second, the atrioventricular (AV) node and the bundle of His traverses 3 to $5 \mathrm{~mm}$ posterior to the anteroseptal commissure. Injury to the node can lead to complete heart block. Third, the right coronary artery (RCA) courses in the right $\mathrm{AV}$ groove posteriorly to the crux of the heart. Although the proximal portion of the RCA is distant from the TA, there is gradual shortening of the distance to $<3 \mathrm{~mm}$ toward the inferior segment of the TA, thereby increasing the risk of RCA injury with transcatheter TV annuloplasty devices.

\section{Transcatheter TV repair therapies}

Annular dilation (increased septal-lateral dimension) and leaflet tethering resulting in malcoaptation is the underlying mechanism of functional (secondary) TR, which accounts for $90 \%$ of the cases in adults (12). Thus, transcatheter TV repair devices are aimed at improving leaflet coaptation either directly by bringing the leaflets together (leaflet/ coaptation devices) or indirectly by repairing the dilated annulus (annuloplasty devices) (Table 1) (37).

\section{Leaflet/coaptation devices}

\section{MitraClip}

The MitraClip (Abbott Vascular, Santa Clara, CA, USA) device is a cobalt chromium and covered-polyester implant with 2 arms that are opened and closed by control mechanisms on the clip delivery system. The clip can be repositioned or removed, and additional clips can be implanted to achieve adequate TR reduction. Two methods to achieve reduction of TR with the MitraClip system have

Table 1 Current devices for transcatheter tricuspid valve repair

\begin{tabular}{|c|c|c|c|c|c|}
\hline Device & Image & Clinical studies & $\begin{array}{c}\text { Procedural } \\
\text { success }\end{array}$ & Mortality & Ongoing studies \\
\hline \multicolumn{6}{|c|}{ Leaflet/coaptation devices } \\
\hline PASCAL & & Case report (18) & - & - & CLASP TR (19) \\
\hline
\end{tabular}

Table 1 (continued) 
Table 1 (continued)

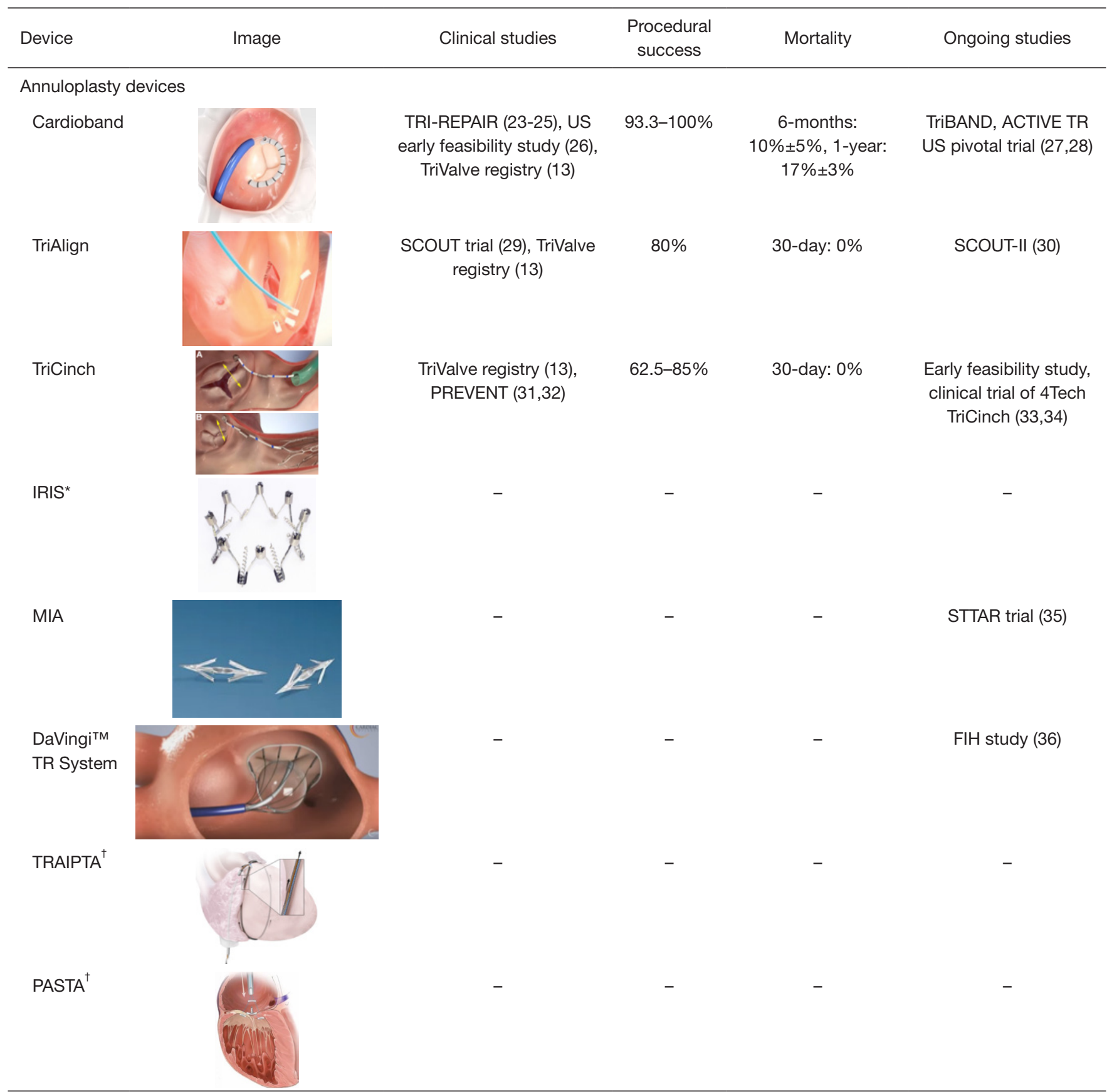

*, the transcatheter delivery system for the tricuspid IRIS is currently under clinical development; ${ }^{\dagger}$, animal studies only. TRILUMINATE, Trial to Evaluate Treatment With Abbott Transcatheter Clip Repair System in Patients With Moderate or Greater Tricuspid Regurgitation; CLASP TR, Edwards PASCAL TrAnScatheter Valve RePair System in Tricuspid Regurgitation Early Feasibility Study; SPACER, Repair of Tricuspid Valve Regurgitation Using the Edwards TricuSPid TrAnsCatheter REpaiR System; TRI-REPAIR, Trlcuspid Regurgitation RePAlr With CaRdioband Transcatheter System; ACTIVE TR, US Edwards Cardioband Tricuspid Valve Reconstruction System Early Feasibility Study; SCOUT, Transcatheter Tricuspid Valve Annuloplasty System for Symptomatic Chronic Functional Tricuspid Regurgitation; PREVENT, Transcatheter Treatment of Tricuspid Valve Regurgitation With the TriCinch System; MIA, minimally invasive annuloplasty; STTAR, Study of Transcatheter Tricuspid Annular Repair; FIH, first in human; TRAIPTA, transatrial intrapericardial tricuspid annuloplasty; PASTA, pledgetassisted suture tricuspid annuloplasty. 
been described: (I) triple-orifice technique (TOT), where clips are placed centrally between the septal and anterior tricuspid leaflet as well as the septal and posterior tricuspid leaflet; and (II) bicuspidization technique (BT), where clips are placed between the septal and anterior tricuspid leaflet (38). Results are comparable with the two techniques; however, the BT is considered technically more feasible and is therefore performed more frequently (38).

The off-label use of the MitraClip system is the most commonly performed transcatheter TV edge-to-edge repair (TTVr) procedure, either for isolated severe TR or combined severe TR and severe mitral regurgitation (MR) (13). Several single-center studies and multicenter registries of patients with severe TR treated with TTVr using the MitraClip system have demonstrated acute procedural success (defined as a 1-grade improvement in TR severity) rates of $>90 \%$. Even a modest improvement in TR severity is associated with significant improvement in NYHA functional class, 6-minute walk distance (6MWD), and quality of life (QoL) up to 1 year (13-16). Mortality rates in various studies were $2.8 \%, 16 \%$, and $37.5 \%$ at 1,6 , and 12 months, respectively $(13,15,16)$. Two recent studies also showed that TTVr is associated with RV reverse remodeling as evidenced by significant reductions in average $\mathrm{RV}$ and $\mathrm{TV}$ dimensions, $\mathrm{RV}$ end-diastolic area, $\mathrm{RV}$ end-systolic area, and septal-lateral TV annulus diameter $(39,40)$. Further, in patients with severe TR and abnormal baseline liver function TTVr was associated with significant reductions in aspartate transaminase and bilirubin (41).

The Trial to Evaluate Treatment With Abbott Transcatheter Clip Repair System in Patients With Moderate or Greater Tricuspid Regurgitation (TRILUMINATE) is an ongoing prospective, single-arm, multicenter study to evaluate the safety and effectiveness of the Tricuspid Valve Repair System (Abbott Vascular, Santa Clara, CA, USA) for treating symptomatic $\geq 2+$ TR in patients currently on medical management and who are deemed appropriate for transcatheter intervention (17).

\section{PASCAL}

The PASCAL Transcatheter Valve Repair System consists of a $10-\mathrm{mm}$ central spacer, which acts as filler in the regurgitant orifice of the valve and is attached to the valve leaflets by two paddles and clasps. The wide spring-loaded paddles ( $25 \mathrm{~mm}$ width in grasping position) and clasps (10 mm length) allow uniform distribution of load across the surface area of the grasped leaflets. The convex curvature of the tip of the paddles reduces tension on the valve leaflets (42).

The first-in-man compassionate use of the Edwards PASCAL transcatheter mitral valve repair system (Edwards Lifesciences, Irvine, CA, USA) in 23 patients with severe MR has been previously reported (42). Fam et al. (18) recently reported a case of an 82-year-old woman with torrential TR and NYHA functional class IV dyspnea, severe fatigue, ascites, and peripheral edema, who underwent successful TTVr using the PASCAL system with reduction of TR to mild, improvement in NYHA functional class to II, resolution of ascites, and improvement in QoL and 6MWD at 1 month. However, further research to assess the safety, efficacy, and durability of this system is needed. The Edwards PASCAL TrAnScatheter Valve RePair System in Tricuspid Regurgitation (CLASP TR) Early Feasibility Study is an ongoing multicenter, prospective, single-arm, non-randomized study to evaluate the safety and performance of the PASCAL system in 15 patients with symptomatic severe functional or degenerative TR (19). The primary outcome measure in this study is freedom from device- or procedure-related adverse events at 30 days. Secondary outcome measures will include NYHA functional class and reduction in TR grade up to 5 years, and 6MWD and health status [Kansas City Cardiomyopathy Questionnaire (KCCQ) and 36-Item Short Form Health Survey (SF-36)] up to 1 year follow-up.

\section{FORMA}

The FORMA system (Edwards Lifesciences, Irvine, CA, USA) is a spacer device (42 $\mathrm{mm}$ length) placed within the TV over a rail that is anchored into the RV myocardium (43). The holes within the spacer shaft allow the spacer to expand passively and act as a surface for valve leaflet coaptation, thereby reducing the effective regurgitant orifice area (EROA). Two coaptation diameter sizes are currently available-12 and $15 \mathrm{~mm}$. The 1 -year clinical and echocardiographic outcomes of the first-in-human, multicenter, compassionate use experience with the FORMA system has been reported (20). At 1 year there were no deaths, significant arrhythmias, device infections, or dislocations. One out of 15 (7\%) patients developed device thrombosis at 1 year. Among the 14 patients with successful device implantation and 1-year follow-up, 79\% were in NYHA functional class I/II $(\mathrm{P}<0.001)$, the average $6 \mathrm{MWD}$ increased by $84 \mathrm{~m}(\mathrm{P}=0.03)$, and the Kansas City Cardiomyopathy Questionnaire (KCCQ) score improved by 18 points $(\mathrm{P}=0.02)$ compared with baseline. Reduction in TR to $\leq$ moderate-to-severe was achieved in $69 \%$ of patients 
by 30 days $(\mathrm{P}=0.001)$ and $46 \%$ by 1 year $(\mathrm{P}=0.01)(20)$.

The safety and efficacy of the FORMA system will be further evaluated in two ongoing studies-the Early Feasibility Study of the Edwards FORMA Tricuspid Transcatheter Repair System, which will enroll 60 participants with the primary outcome measure of procedural success (defined as device success and freedom from device or procedure related severe adverse events) at 30 days, and the Repair of Tricuspid Valve Regurgitation Using the Edwards TricuSPid TrAnsCatheter REpaiR System (SPACER) trial, which will enroll 78 participants and examine the primary endpoint of cardiac mortality of the as-treated cohort at 30 days compared with a literaturederived performance goal based on high-risk surgical outcomes for TV repair/replacement $(21,22)$.

\section{Annuloplasty devices}

\section{Cardioband TV reconstruction system}

The Cardioband (Edwards Lifesciences, Irvine, CA, USA) is a polyester sleeve with radiopaque markers spaced $8 \mathrm{~mm}$ apart. The sleeve contains a pre-mounted contraction wire connected to an adjusting spool (44). Twelve to seventeen anchors are implanted through the sleeve. Once the last anchor is delivered, the implant is then contracted using a size adjustment tool. The Cardioband has been implanted under compassionate use in 5 patients with functional TR with satisfactory results- $23 \%$ to $45 \%$ reduction of the TA diameter and $50 \%$ to $70 \%$ reduction of the EROA without any procedure-related serious adverse events (45).

The Cardioband system received Conformité Européene (CE) mark approval in April 2018 based on results of the TrIcuspid Regurgitation RePAIr With CaRdioband Transcatheter System (TRI-REPAIR) study, which enrolled 30 patients with NYHA functional class $\geq$ II and moderate or greater functional TR $(23,24,46)$. Technical success was $100 \%$. At 6 months, the Cardioband system resulted in average reductions of annual septolateral diameter of $9 \%(\mathrm{P}<0.01)$, proximal isovelocity surface area EROA of $50 \%(\mathrm{P}<0.001)$, and mean vena contracta width of $28 \%(\mathrm{P}<0.001)$ compared with baseline $(24)$. LVSV increased from $59.2 \pm 19.7$ to $64.5 \pm 12.1 \mathrm{~mL}(\mathrm{P}=0.07)$ and $61.1 \pm 17.7$ to $64.6 \pm 11.7 \mathrm{~mL}(\mathrm{P}=0.26)$ after 30 days and 6 months, respectively (24). The mean $6 \mathrm{MWD}$ increased by $60 \mathrm{~m}(\mathrm{P}=0.004)$, KCCQ score improved by 24 points $(\mathrm{P}<0.001)$, and $76 \%$ of patients improved by at least 1 NYHA functional class with $88 \%$ in NYHA functional class I or II at 6 months. The echocardiographic and clinical improvements were sustained at 1 year follow-up (25). The primary safety endpoint of major serious adverse events (a composite of death, myocardial infarction, cardiac tamponade, device-related cardiac surgery, and stroke) occurred in $13.3 \%$ of patients at 30 days (24). All-cause mortality rate was $10 \% \pm 5 \%$ at 6 months and $17 \% \pm 3 \%$ at 1 year $(24,25)$. The Transcatheter Repair of Tricuspid Regurgitation With Cardioband TR Sytem Post Market Study (TriBAND) is a European, prospective, single-arm, multicenter post-market follow-up study to assess the safety and efficacy of the Edwards Cardioband TR system in patients with symptomatic, chronic moderate to severe $(\geq 2+)$ functional TR (27). The study is expected to enroll 150 patients who will be followed up at 30 days, 6 months, and annually up to 5 years.

Thirty-day results of the US Edwards Cardioband Tricuspid Valve Reconstruction System Early Feasibility Study, which enrolled 15 patients with symptomatic chronic functional TR of moderate or greater severity, were recently presented (26). The mean age of patients was $80 \pm 8$ years, $73 \%$ were women, and $73 \%$ were in NYHA functional class III or IV at baseline (26). Technical success was $93.3 \%$. At 30 days, the Cardioband system resulted in average reductions of annual septolateral diameter of $14 \%(\mathrm{P}<0.001)$, proximal isovelocity surface area EROA of $40 \%(\mathrm{P}<0.018)$, and mean vena contracta width of $28 \%$ $(\mathrm{P}<0.001)$ compared with baseline $(26)$. The mean 6MWD increased by $7 \mathrm{~m}(\mathrm{P}=\mathrm{NS})$, KCCQ score improved by 13 points $(\mathrm{P}=0.015)$, and $64 \%$ of patients were in NYHA functional class I or II at 30 days $(\mathrm{P}=0.047)(26)$. Thirtyday mortality was $0 \%$. Serious adverse events included RCA constriction in $1(6.7 \%)$ patient and severe bleeding in 5 (33.3\%) patients (26). The ACTIVE TR US Pivotal Trial is expected to begin in late 2019 (28).

\section{TriAlign}

The TriAlign device (Mitralign Inc., Tewksbury, MA, USA) is a transcatheter suture-based tricuspid annuloplasty system that attempts to replicate the results of the current modified Kay procedure, which has shown long-term efficacy similar to those of other surgical TV repair methods (47-49). The posterior leaflet is plicated using 2 pledgets that are positioned at the anteroposterior and septal posterior commissure and then sutured together using the dedicated plication lock device. A distance of $25-28 \mathrm{~mm}$ between the 2 pledgets is recommended (50). In case of suboptimal results, a second pair of pledgets can be implanted to obtain a consistent reduction in annular dimensions. 
The feasibility and safety of the TriAlign system was evaluated in 15 patients with NYHA functional class $\geq$ II and moderate or greater functional TR enrolled in the Transcatheter Tricuspid Valve Annuloplasty System for Symptomatic Chronic Functional Tricuspid Regurgitation (SCOUT) trial (29). Technical success rate at 30 days was $80 \%$. Three patients had single-pledget annular detachments that did not necessitate reintervention. In the remaining 12 patients, the TriAlign system significantly reduced TA diameter and EROA, with significant increase in LVSV. In the intention-to-treat cohort, there were significant improvements in NYHA functional class $(\geq 1$ class, $\mathrm{P}=0.001)$, Minnesota Living with Heart Failure Questionnaire (MLHFQ) $(47.4 \pm 17.6$ to $20.9 \pm 14.8$; $\mathrm{P}<0.001)$, and $6 \mathrm{MWD}(245.2 \pm 110.1$ to $298.0 \pm 107.6 \mathrm{~m}$; $\mathrm{P}=0.008$ ) (29). Data from the TriValve Registry on 18 patients who underwent TV repair with the TriAlign system demonstrated procedural success rate of $69.2 \%$ and zero deaths at 30 days. The Safety and Performance of the TriAlign Transcatheter Tricuspid Valve Annuloplasty System for Symptomatic Chronic Functional Tricuspid Regurgitation (SCOUT-II) is an ongoing prospective, single-arm, multicenter, open-label study that will enroll up to 60 patients from up to 15 sites in Europe and US (30). The primary endpoint is 30 -day all-cause mortality.

\section{TriCinch}

The TriCinch system (4Tech Cardio Ltd, Galway, Ireland) reproduces the Kay procedure by cinching at the anteroposterior commissure, thus reducing septolateral dimensions (45). The TriCinch system consists of two components: (I) a stainless steel corkscrew implant, to be placed in the anterior TA, in proximity to the anteroposterior commissure, and (II) a self-expanding nitinol stent that is deployed below the hepatic region of the inferior vena cava (51). The safety and efficacy of this device was investigated in the Transcatheter Treatment of Tricuspid Valve Regurgitation With the TriCinch System $^{\text {TM }}$ (PREVENT) first-in-man feasibility study (31). Among the 24 patients treated, procedural success rate was $85 \%$. Reasons for unsuccessful procedure included hemopericardium requiring interruption of the procedure $(n=2)$ and late detachment of the anchor $(n=4)(32)$. Among the 14 patients who underwent TV repair with the TriCinch system in the TriValve Registry, procedural success rate was $62.5 \%$ with zero deaths at 30 days (13). The safety and performance of the TriCinch Coil System in symptomatic patients with moderate to severe TR
$(2+$ to $4+)$ and annual diameter $\geq 40 \mathrm{~mm}$ confirmed by echocardiography will be further evaluated in two open label, single arm studies-the Early Feasibility Study of the Percutaneous 4Tech TriCinch Coil Tricuspid Valve Repair System and the Clinical Trial Evaluation of the Percutaneous 4Tech TriCinch Coil Tricuspid Valve Repair System $(33,34)$. The primary outcome measure in both studies will be 30 -day all-cause mortality.

\section{IRIS}

The IRIS transcatheter annuloplasty ring (Millipede, Inc., Santa Rosa, CA, USA) is a complete semi-rigid ring that is placed in the supra-annular position and is then anchored and cinched thereby reducing the annular size and TR. The IRIS implant consists of three components: a frame made of nitinol formed into a ring, anchors that engage the annular tissue, and collars that reduce the diameter of the frame to achieve proper valve leaflet coaptation. The IRIS has 2 distinct advantages-first, the ring is completely repositionable and adjustable prior to final deployment and second, the ring preserves the native anatomy without precluding future transcatheter options such as transcatheter edge-to-edge repair. A transcatheter delivery system for the tricuspid IRIS is currently under clinical development.

\section{MIA}

The minimally invasive annuloplasty (MIA) device (Micro Interventional Devices, Inc., Newton, PA, USA) is a compliant, self-tensioning implant incorporating the company's proprietary PolyCor ${ }^{\mathrm{TM}}$ anchors and MyoLast ${ }^{\mathrm{TM}}$ thermoplastic elastomer that reduces TA dimension without sutures or other intervention. The safety and efficacy of the MIA device will be evaluated in 40 patients with functional TR in the Study of Transcatheter Tricuspid Annular Repair (STTAR) trial (35).

\section{DaVingi ${ }^{\mathrm{TM}}$ TR system}

The DaVingi ${ }^{\mathrm{TM}}$ TR system (Cardiac Implants LLC, Wilmington, DE, USA) is a transcatheter device designed to deliver an annuloplasty ring on the atrial side of the TV using right heart catheterization through the right internal jugular vein (36). The annuloplasty ring is a small multielement ring, consisting of an outer fabric layer, a pre-set stakes array, and an internal adjustment cord that can be adjusted at a later stage after the outer layer of the ring and stakes are encapsulated in new tissue growth. The safety and performance of the system will be evaluated in 15 patients with severe TR in the first in human study to assess 
safety and performance of the DaVingi ${ }^{\mathrm{TM}} \mathrm{TR}$ system in the treatment of patients with functional TR (36).

\section{TRAIPTA}

Transatrial intrapericardial tricuspid annuloplasty (TRAIPTA) is a novel experimental TTVr system in which the right atrial appendage (RAA) is punctured from within to access the pericardium, which then allows a circumferential implant to be delivered along the AV groove within the pericardial space (52). The implant exerts compressive force over the TA. Tension on the implant can be adjusted to modify TA geometry and reduce TR. The RAA puncture is sealed using nitinol closure devices. In a preclinical animal study, TRAIPTA reduced the tricuspid septolateral and anteroposterior dimensions, the annular area and perimeter, by $49 \%, 31 \%, 59 \%$, and $24 \%(\mathrm{P}<0.001)$, respectively (52). Small pericardial effusions were observed immediately post-procedure, but resolved completely at follow-up. In 4 animals with functional TR, severity of TR by intracardiac echocardiography was reduced (52). This technique requires the pericardial space to be free of adhesions, thus precluding its use in patients with previous pericardiotomy or pericarditis.

\section{PASTA}

Pledget-assisted suture tricuspid annuloplasty (PASTA) is a novel experimental transcatheter technique to create a double-orifice TV (53). This technique is based on the Hetzer's double orifice suture technique, which has been performed in more than 90 patients with severe TR with no reoperation after 8.7 years (54). In PASTA, pledgeted sutures are delivered via a transcatheter approach to appose septal and lateral targets on the TA, thereby reducing the TV orifice. In a preclinical animal study, PASTA successfully reduced annular and chamber dimensions and TR (53). Four animals had procedure-related complications (leaflet tearing, chord entrapment, leaflet entrapment, transient AV node block, and ventricular fibrillation).

\section{Summary}

Despite the high prevalence of TR and its association with increased mortality, $<8,000 \mathrm{TV}$ surgeries (repair or replacement) are performed annually, and the majority of patients are managed medically in the absence of another indication for cardiac surgery (5). Isolated TV surgery is rarely performed ( 500 per year) (9). To address this unmet clinical need, several less invasive transcatheter
TV therapies have emerged as an alternative to surgery in extreme- and high-risk patients with severe functional TR (37). The short- and mid-term data on the safety and efficacy of various transcatheter TV therapies are encouraging. Procedural and clinical outcomes are expected to improve in the coming years with technological advancement, newer device iterations, and increased experience in this field. Appropriate patient selection, optimal timing of intervention, and evaluation of long-term outcomes and device durability will be key in ongoing and future studies.

\section{Acknowledgments}

None.

\section{Footnote}

Conflicts of Interest: The authors have no conflicts of interest to declare.

Etbical Statement: The authors are accountable for all aspects of the work in ensuring that questions related to the accuracy or integrity of any part of the work are appropriately investigated and resolved.

\section{References}

1. Demir OM, Regazzoli D, Mangieri A, et al. Transcatheter Tricuspid Valve Replacement: Principles and Design. Front Cardiovasc Med 2018;5:129.

2. Singh JP, Evans JC, Levy D, et al. Prevalence and clinical determinants of mitral, tricuspid, and aortic regurgitation (the Framingham Heart Study). Am J Cardiol 1999;83:897-902.

3. Topilsky Y, Maltais S, Medina IJ, et al. Burden of Tricuspid Regurgitation in Patients Diagnosed in the Community Setting. JACC Cardiovasc Imaging 2019;12:433-42.

4. Nath J, Foster E, Heidenreich PA. Impact of tricuspid regurgitation on long-term survival. J Am Coll Cardiol 2004:43:405-9.

5. Stuge O, Liddicoat J. Emerging opportunities for cardiac surgeons within structural heart disease. J Thorac Cardiovasc Surg 2006;132:1258-61.

6. Vassileva CM, Shabosky J, Boley T, et al. Tricuspid valve surgery: the past 10 years from the Nationwide Inpatient Sample (NIS) database. J Thorac Cardiovasc Surg 
2012;143:1043-9.

7. Kim JB, Jung SH, Choo SJ, et al. Clinical and echocardiographic outcomes after surgery for severe isolated tricuspid regurgitation. J Thorac Cardiovasc Surg 2013;146:278-84.

8. Kwon DA, Park JS, Chang HJ, et al. Prediction of outcome in patients undergoing surgery for severe tricuspid regurgitation following mitral valve surgery and role of tricuspid annular systolic velocity. Am J Cardiol 2006;98:659-61.

9. Zack CJ, Fender EA, Chandrashekar P, et al. National Trends and Outcomes in Isolated Tricuspid Valve Surgery. J Am Coll Cardiol 2017;70:2953-60.

10. Rodés-Cabau J, Hahn RT, Latib A, et al. Transcatheter Therapies for Treating Tricuspid Regurgitation. J Am Coll Cardiol 2016;67:1829-45.

11. Dahou A, Levin D, Reisman M, et al. Anatomy and Physiology of the Tricuspid Valve. JACC Cardiovasc Imaging 2019;12:458-68.

12. Fender EA, Zack CJ, Nishimura RA. Isolated tricuspid regurgitation: outcomes and therapeutic interventions. Heart 2018;104:798-806.

13. Taramasso M, Alessandrini H, Latib A, et al. Outcomes After Current Transcatheter Tricuspid Valve Intervention: Mid-Term Results From the International TriValve Registry. JACC Cardiovasc Interv 2019;12:155-65.

14. Nickenig G, Kowalski M, Hausleiter J, et al. Transcatheter Treatment of Severe Tricuspid Regurgitation With the Edge-to-Edge MitraClip Technique. Circulation 2017;135:1802-14.

15. Orban M, Besler C, Braun D, et al. Six-month outcome after transcatheter edge-to-edge repair of severe tricuspid regurgitation in patients with heart failure. Eur J Heart Fail 2018;20:1055-62.

16. Braun D, Nabauer M, Orban M, et al. One-year results of transcatheter treatment of severe tricuspid regurgitation using the edge-to-edge repair technique. EuroIntervention 2018;14:e413-5.

17. NCT03227757 Evaluation of Treatment With Abbott Transcatheter Clip Repair System in Patients With Moderate or Greater Tricuspid Regurgitation (TRILUMINATE). C. 12-18-2018. 1-18-2019.

18. Fam NP, Ho EC, Zahrani M, et al. Transcatheter Tricuspid Valve Repair With the PASCAL System. JACC Cardiovasc Interv 2018;11:407-8.

19. NCT03745313 Edwards PASCAL TrAnScatheter Valve RePair System in Tricuspid Regurgitation (CLASP TR) Early Feasibility Study. 5-20-2019. ClinicalTrials.gov.
6-4-2019.

20. Perlman G, Praz F, Puri R, et al. Transcatheter Tricuspid Valve Repair With a New Transcatheter Coaptation System for the Treatment of Severe Tricuspid Regurgitation: 1-Year Clinical and Echocardiographic Results. JACC Cardiovasc Interv 2017;10:1994-2003.

21. NCT02471807 Early Feasibility Study of the Edwards FORMA Tricuspid Transcatheter Repair System. 3-222018. ClinicalTrials.gov. 1-18-2019.

22. NCT02787408 The SPACER Trial - Repair of Tricuspid Valve Regurgitation Using the Edwards TricuSPid TrAnsCatheter REpaiR System. 12-26-2017. ClinicalTrials.gov. 1-18-2019.

23. Nickenig G. TRI-REPAIR: 30-Day Outcomes of Transcatheter TV Repair in Patients With Severe Secondary Tricuspid Regurgitation. Transcatheter Cardiovascular Therapeutics 2017. 11-2-2017. Denver, CO. 11-2-2017.

24. Nickenig G, Weber M, Schueler R, et al. 6-Month Outcomes of Tricuspid Valve Reconstruction for Patients With Severe Tricuspid Regurgitation. J Am Coll Cardiol 2019;73:1905-15.

25. Kreidel F. 1-Year Outcomes of the TRI-REPAIR Study Assessing Cardioband Tricuspid Valve Reconstruction System for Patients With Functional Tricuspid Regurgitation. www.tctmd.com. 6-14-2019. 6-16-2019.

26. Lim S. Results From the Early Feasibility Study of Cardioband Tricuspid System for Functional Tricuspid Regurgitation. www.tctmd.com. 6-14-2019. 6-16-2019.

27. NCT03779490 Transcatheter Repair of Tricuspid Regurgitation With Cardioband TR Sytem Post Market Study (TriBAND): A European Prospective, Multicenter Post-market Clinical Follow-up Study to Assess Transcatheter Tricuspid Valve Repair With Edwards Cardioband TR System in Patients With Symptomatic, Chronic Functional Tricuspid Regurgitation. 12-18-2018. ClinicalTrials.gov. 7-4-2019.

28. Zovighian BV. Transcatheter Mitral and Tricuspid Therapies. 12-5-2018. Edwards Lifesciences. 6-4-2019.

29. Hahn RT, Meduri CU, Davidson CJ, Early Feasibility Study of a Transcatheter Tricuspid Valve Annuloplasty: SCOUT Trial 30-Day Results. J Am Coll Cardiol 2017;69:1795-806.

30. NCT03225612 Safety and Performance of the Trialign Percutaneous Tricuspid Valve Annuloplasty System (PTVAS) (SCOUT-II). 2-7-2018. ClinicalTrials.gov. 1-18-2019.

31. NCT02098200 Percutaneous Treatment of Tricuspid 
Valve Regurgitation With the TriCinch System ${ }^{\mathrm{TM}}$ (PREVENT). 3-14-2018. ClinicalTrials.gov. 1-18-2019.

32. Giannini F, Colombo A. Percutaneous treatment of tricuspid valve in refractory right heart failure. Eur Heart J Suppl 2019;21:B43-7.

33. NCT03632967 Early Feasibility Study of the Percutaneous 4Tech TriCinch Coil Tricuspid Valve Repair System. 4-30-2019. ClinicalTrials.gov. 7-4-2019.

34. NCT03294200 Clinical Trial Evaluation of the Percutaneous 4Tech TriCinch Coil Tricuspid Valve Repair System. 4-30-2019. ClinicalTrials.gov. 7-4-2019.

35. First Clinical Tricuspid Bicuspidization Procedure Completed Using Micro Interventional Devices' MIA Technology. 9-7-2017. Cardiac Interventions TODAY.

36. NCT03700918 A First in Human Study to Assess Safety and Performance of the DaVingi ${ }^{\text {TM }}$ TR System in the Treatment of Patients With Functional Tricuspid Regurgitation. 10-15-2018. ClinicalTrials.gov. 7-4-2019.

37. Kolte D, Elmariah S. Transcatheter Tricuspid Valve Therapy. Curr Treat Options Cardiovasc Med 2019;21:26.

38. Braun D, Orban M, Orban M, et al. Transcatheter Edgeto-Edge Repair for Severe Tricuspid Regurgitation Using the Triple-Orifice Technique Versus the Bicuspidalization Technique. JACC Cardiovasc Interv 2018;11:1790-2.

39. Orban M, Braun D, Deseive S, et al. Transcatheter Edgeto-Edge Repair for Tricuspid Regurgitation Is Associated With Right Ventricular Reverse Remodeling in Patients With Right-Sided Heart Failure. JACC Cardiovasc Imaging 2019;12:559-60.

40. Ledwoch J, Fellner C, Poch F, et al. Reverse Cardiac Remodeling After Transcatheter Treatment of Severe Tricuspid Regurgitation Using the Edge-to-Edge MitraClip Technique. J Invasive Cardiol 2019;31:89-93.

41. Karam N, Braun D, Mehr M, et al. Impact of Transcatheter Tricuspid Valve Repair for Severe Tricuspid Regurgitation on Kidney and Liver Function. JACC Cardiovasc Interv 2019;12:1413-20.

42. Praz F, Spargias K, Chrissoheris M, et al. Compassionate use of the PASCAL transcatheter mitral valve repair system for patients with severe mitral regurgitation: a multicentre, prospective, observational, first-in-man study.
Lancet 2017;390:773-80.

43. Puri R, Rodes-Cabau J. The FORMA Repair System. Interv Cardiol Clin 2018;7:47-55.

44. Messika-Zeitoun D, Nickenig G, Latib A, et al. Transcatheter mitral valve repair for functional mitral regurgitation using the Cardioband system: 1 year outcomes. Eur Heart J 2019;40:466-72.

45. Mangieri A, Lim S, Rogers JH, et al. Percutaneous Tricuspid Annuloplasty. Interv Cardiol Clin 2018;7:31-6.

46. Edwards Granted CE Mark For First Transcatheter Tricuspid Therapy. 4-30-2018. Irvine, CA, Edwards Lifesciences, Inc. 1-18-2019.

47. Schofer J, Bijuklic K, Tiburtius C, et al. First-in-human transcatheter tricuspid valve repair in a patient with severely regurgitant tricuspid valve. J Am Coll Cardiol 2015;65:1190-5.

48. Kay JH, Maselli-Campagna G, Tsuji KK. Surgical treatment of tricuspid insufficiency. Ann Surg 1965;162:53-8.

49. Ghanta RK, Chen R, Narayanasamy N, et al. Suture bicuspidization of the tricuspid valve versus ring annuloplasty for repair of functional tricuspid regurgitation: midterm results of 237 consecutive patients. J Thorac Cardiovasc Surg 2007;133:117-26.

50. Besler C, Meduri CU, Lurz P. Transcatheter Treatment of Functional Tricuspid Regurgitation Using the Trialign Device. Interv Cardiol 2018;13:8-13.

51. Rosser BA, Taramasso M, Maisano F. Transcatheter interventions for tricuspid regurgitation: TriCinch (4Tech). EuroIntervention 2016;12:Y110-2.

52. Rogers T, Ratnayaka K, Sonmez M, et al. Transatrial intrapericardial tricuspid annuloplasty. JACC Cardiovasc Interv 2015;8:483-91.

53. Khan JM, Rogers T, Schenke WH, et al. Transcatheter pledget-assisted suture tricuspid annuloplasty (PASTA) to create a double-orifice valve. Catheter Cardiovasc Interv 2018;92:E175-84.

54. Hetzer R, Javier M, Delmo Walter EM. The DoubleOrifice Valve Technique to Treat Tricuspid Valve Incompetence. J Heart Valve Dis 2016;25:66-71.
Cite this article as: Kolte D, Elmariah S. Current state of transcatheter tricuspid valve repair. Cardiovasc Diagn Ther 2020;10(1):89-97. doi: 10.21037/cdt.2019.09.11 